\title{
Pharmacogenetic polymorphism as an independent risk factor for frequent hospitalizations in older adults with polypharmacy: a pilot study
}

\author{
This article was published in the following Dove Press journal: \\ Pharmacogenomics and Personalized Medicine \\ 14 October 2016 \\ Number of times this article has been viewed
}

\author{
Joseph Finkelstein' \\ Carol Friedman' \\ George Hripcsak' \\ Manuel Cabrera ${ }^{2}$ \\ 'Department of Biomedical \\ Informatics, ${ }^{2}$ Department of \\ Medicine, Columbia University \\ College of Physicians and Surgeons, \\ New York, NY, USA
}

Correspondence: Joseph Finkelstein Department of Biomedical Informatics, Columbia University College of Physicians and Surgeons, 622 West 168th Street, PH-20, Room 40IA, New York, NY 10032, USA

Tel +l 9174773909

Email jf193@cumc.columbia.edu
Abstract: Pharmacogenetic testing identifies genetic biomarkers that are predictive of individual sensitivity to particular drugs. A significant proportion of medications that are widely prescribed for older adults are metabolized by enzymes that are encoded by highly polymorphic genes. Pharmacogenetic testing is increasingly used to optimize the medication regimen; however, its potential in older adults with polypharmacy has not been systematically explored. Following the initial case-series study, this study hypothesized that frequently hospitalized older adults with polypharmacy have higher frequency of pharmacogenetic polymorphism as compared to older adults with polypharmacy who are rarely admitted to a hospital. To test this hypothesis, a nested case-control study was conducted with pharmacogenetic polymorphism as an exposure and hospitalization rate as an outcome. In this study, frequently hospitalized older adults $(\geq 65$ years of age) with polypharmacy were matched with rarely hospitalized older adults with polypharmacy by age, gender, race, ethnicity, and chronic disease score. Average age and number of prescription drugs did not differ in cases and controls (77.2 \pm 5.0 and $78.3 \pm 5.1$ years, $14.3 \pm 5.3$ and $14.0 \pm 2.9$ medications, respectively). No statistically significant difference in sociodemographic, clinical, and behavioral characteristics that are known to affect hospitalization risk was found between the cases and controls. Major pharmacogenetic polymorphism defined as presence of at least one allelic combination resulting in poor or rapid metabolizer status was identified in all the cases. No major pharmacogenetic polymorphisms were detected in controls. Based on the exact McNemar's test, the difference in major pharmacogenetic polymorphism frequency between cases and controls was statistically significant $(p<0.05)$. In $50 \%$ of cases, more than one major pharmacogenetic polymorphism was found. The frequency of CYP2C19 rapid metabolizer, CYP3A4/5 poor metabolizer, VKORC1 low sensitivity, and CYP2D6 rapid metabolizer status in cases was $67 \%, 33 \%, 33 \%$, and $17 \%$, respectively, which significantly exceeded respective prevalence in general population. The mean number of major gene-drug interactions found in cases was $2.8 \pm 2.2$, whereas no major drug-gene interactions were identified in controls. The difference in the number of major drug-gene interactions between cases and controls was statistically significant $(p<0.05)$. The pilot data supported the hypothesis that pharmacogenetic polymorphism may represent an independent risk factor for frequent hospitalizations in older adults with polypharmacy. Due to small sample size, the results of this proof-of-concept study cannot be conclusive. Further work on the utility of pharmacogenetic testing for optimization of medication regimens in this vulnerable group of older adults is warranted.

Keywords: urgent care utilization, risk factors, personalized medicine, elderly, case-control study 


\section{Background}

Advances in medical sciences and discovery of new medications have significantly extended life expectancy and led to a drastic increase of people living with multiple comorbidities. ${ }^{1}$ Rapid population aging in developed countries has resulted in a growing demand for medical services and a significant increase in health care expenditures. Urgent care utilization among older adults represents a significant portion of annual expenditures on hospital care. Older adults have more hospital stays than any other age group. ${ }^{2}$ While older adults comprise $\sim 12 \%$ of the US population, they account for onethird of all hospitalizations in the US, totaling $\sim$ \$329 billion or $44 \%$ of national hospital expenditures. ${ }^{2}$ Multiple risk factors for hospitalization of older adults have been reported in various studies. ${ }^{3,4}$ They include age, gender, education, social status, presence of geriatric syndromes, comorbidity scores, history of urgent care utilization, and number of medications. ${ }^{4}$ Effective approaches are urgently needed to address the growing health care needs of an aging population while also improving quality of medical care and decreasing its cost.

Older adults with polypharmacy exhibit higher rates of hospitalizations and adverse drug events (ADEs). ${ }^{5}$ In a cohort of 887,165 older adults, $39.4 \%$ had polypharmacy defined as simultaneous use of $\geq 5$ medications. ${ }^{6}$ Among the known risk factors of urgent care utilization, multiple medication regimen or polypharmacy has been consistently identified as one of the strongest modifiable predictors of hospitalization. ${ }^{5}$ A recent prospective cohort study demonstrated that older adults with polypharmacy had $81 \%$ higher risk for 1-year hospitalizations than older adults without polypharmacy. ${ }^{7}$ Another study in older adults found that a patient taking more than 5 medications was almost 4 times more likely to be hospitalized because of ADE. ${ }^{8}$ The US health care system spent $\sim \$ 300$ billion on prescription medications in 2014 with an estimated incremental increase of $6.3 \%$ spending annually over the next decade. ${ }^{9}$ This economic problem is exacerbated by a growing aging population accompanied by relatively disjointed prescribing behaviors and poor patient monitoring. ${ }^{10}$ Modern precision medicine approaches offer powerful tools to optimize medication regimens; however, potential impact of these tools in older adults with polypharmacy has not been fully appreciated yet. ${ }^{11}$

Providing precision medicine means to prescribe personalized treatment regimens based on individual genetic variations. ${ }^{12}$ Pharmacogenetic testing has been increasingly used to identify genetic biomarkers that predict individual sensitivity to particular drugs. ${ }^{13}$ Polymorphism of genes encoding cytochrome P450 (CYP) enzymes has been the major target of pharmacogenetics since CYP enzymes are involved in the metabolism of $>70 \%$ of all prescribed drugs. ${ }^{14}$ Particular attention in pharmacogenetic testing has been devoted to CYP2D6, CYP2C19, CYP2C9, and CYP $3 A 4 / 5$ genes as they encode the most common CYP enzymes involved in drug metabolism and are highly polymorphic. ${ }^{15}$ The various allele combinations of these genes result in a continuum of drug metabolizing activity and the corresponding drug bioavailability. ${ }^{14}$ Individual drug metabolizing activity for corresponding substrates varies from little or no activity (poor metabolizer phenotype), reduced function (intermediate metabolizer phenotype), to "wild-type" activity (extensive metabolizer phenotype), and increased enzyme expression in the case of gene duplication (rapid metabolizer phenotype). ${ }^{16}$ For example, CYP2D6 genotype-stratified pharmacokinetic studies of carvedilo $1^{17}$ and atomoxetine ${ }^{18}$ demonstrated nearly 30 -fold variations in systemic exposure across study cohort represented by a spectrum of subjects from poor to rapid metabolizers. Recent studies also showed an important role of other polymorphisms impacting drug activity such as VCORC1 affecting sensitivity to warfarin. ${ }^{19}$ Previous studies have mainly focused on a single gene-drug interaction and provided conflicting evidence of the clinical utility of pharmacogenetic testing. ${ }^{20}$ Further evidence is necessary to demonstrate the potential of pharmacogenetic testing to improve quality of care for older adults with polypharmacy.

In the recent case series study, it was found that frequently hospitalized older adults with polypharmacy exhibited a very high frequency of pharmacogenetic polymorphisms, whereas no major pharmacogenetic polymorphisms were found in older adults with polypharmacy who were rarely hospitalized. ${ }^{21}$ Provided that all patients had similar comorbidities and disease severity, were receiving guideline-concordant care, and were adherent to their treatment regimen, the study hypothesized that the potential source of wide disparity in hospitalization rates in these patients could be due to different efficacies of drug therapy, caused by differences in individual response to medication regimen. This hypothesis is consistent with recent studies that demonstrated that hospitalization rates may vary widely in older adults even though they have a similar level of disease severity ${ }^{22}$ and that older adults with polypharmacy who were treated according to a pharmacogenetics-guided treatment plan had a significant decrease in urgent care utilization. ${ }^{23}$ However, no systematic comparison of the frequency of pharmacogenetic polymorphisms in older adults with polypharmacy as related to their hospitalization rate has been performed. This study is designed to address this gap in our knowledge. The primary hypothesis to be tested 
in this study is that frequently hospitalized older adults with polypharmacy have a higher frequency of pharmacogenetic polymorphisms as compared to older adults with polypharmacy who are rarely admitted to the hospital.

An enormous burden is imposed on health care because of prescribing inappropriate medication, particularly in the context of older adults and polypharmacy. The overall objective of this pilot project is to explore the potential association between the presence of pharmacogenetic polymorphisms and high hospitalization rates in older adults with polypharmacy using a nested case-control design. Establishing such an association will support a broader introduction of personalized medicine in the care of older adults with polypharmacy.

\section{Methods}

\section{Study design}

The primary goal of this project was to assess whether pharmacogenetic polymorphism is an independent risk factor for frequent hospitalizations (FHs) in older adults with polypharmacy. To address this goal, a comprehensive conceptual framework containing previously described hospitalization risk factors ${ }^{4,24}$ was employed in this study (Figure 1). The framework accounted for major hospitalization risk factors in older adults comprising patient characteristics from clinical, demographic, psycho-behavioral, and social profiles.

In addition to currently accepted risk factors, the framework included a new factor, pharmacogenetic polymorphism, which was not yet systematically assessed as a risk factor for FH in older adults with polypharmacy. Pharmacogenetic polymorphism means the presence of any genetic polymorphism that affects pharmacokinetics or pharmacodynamics of currently prescribed medications.

In order to test the main study hypothesis, a nested case-control study was conducted in older adults with polypharmacy. The study design is presented in Figure 2. The study cohort consisted of older adults with polypharmacy and history of cardiovascular disease to ensure homogeneity

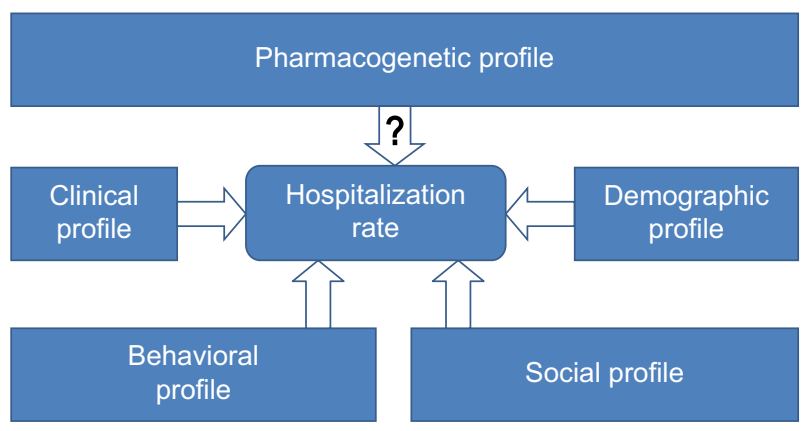

Figure I Hospitalization risk factors.
Cohort

Older adults with polypharmacy and CVD

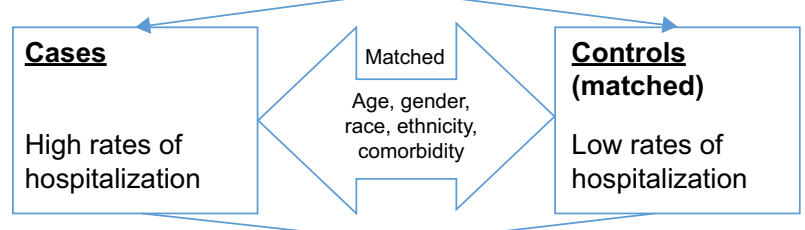

\section{Analysis}

Comparison of PGx frequency in cases and controls

Figure 2 Study design.

Abbreviations: CVD, cardiovascular disease; PGx, pharmacogenetic.

of the study sample. The cases were presented by eligible individuals with the history of FH. The controls included eligible patients with infrequent hospitalizations (IHs) randomly drawn from the study cohort, based on case-control matching criteria. On the basis of a previous work, ${ }^{22}$ cases $(\mathrm{FH})$ were defined as individuals who were hospitalized at least 3 times during the past 2 years. The controls were matched with the cases by age group, gender, race, ethnicity, and chronic disease score (CDS). Subject characteristics were ascertained by a manual chart review supplemented by the patients' self-reports and data from clinical data warehouse.

\section{Study cohort}

The study cohort was chosen on the basis of relevance to the aims of this study and existing evidence on the potential association between the exposure variable (pharmacogenetic polymorphism) and the study outcome (hospitalization rate). The inclusion criteria for the study cohort were: 1) physician diagnosis of chronic cardiovascular condition (congestive heart failure, coronary atherosclerosis, cardiac dysrhythmias, and history of acute coronary syndromes); 2) drug regimen consisting of 5 or more prescription medications; and 3) age $\geq 65$ years of age. Patients who satisfied at least one item from the following exclusion criteria list did not take part in the study: 1) previously having pharmacogenetic testing; 2) a diagnosis of current malabsorption syndrome; 3) currently receiving treatment for or diagnosed with cancer; 4) current diagnosis of malnourishment; 5) a history of organ transplant; 6) receiving intravenous antibiotics or immunosuppressant medications; and 7) chronic liver disease. The exclusion criteria were chosen to minimize bias caused by other factors which may potentially affect drug metabolism in addition to pharmacogenetic polymorphism such as liver disease, malnutrition, or malabsorption. Overall, 6 cases and 6 controls were enrolled in this pilot study. All participants provided written informed consent. 


\section{Sociodemographic profile}

The demographic profile included age, gender, race, ethnicity, income, and number of years spent in school. Age group, gender (female, male), race (Blacks, Whites, Asians, other), and ethnicity (Hispanic, non-Hispanic) were used to match cases and controls. The social profile consisted of variables that were associated with higher hospitalization risk including marital status, presence of caregiver, insurance coverage, and number of people in the household.

\section{Clinical and behavioral profile}

The clinical profile was ascertained on the basis of a comprehensive review of disease history of the patients and comprised hospital utilization over the last 5 years, comorbidities, disease severity, medication regimen, body mass index (BMI), and geriatric syndromes including frailty. Overall disease severity was gauged using the CDS which was developed as an integrative indicator of illness severity in people with multiple comorbidities. ${ }^{25} \mathrm{CDS}$ accounts for the number of different chronic diseases, complexity of medication regimen, and presence of potentially life-threatening or progressive diseases. ${ }^{26}$ In several studies, CDS was shown to predict hospitalization and mortality in the following year. ${ }^{27,28} \mathrm{CDS}$ was found to be an effective means to control for confounding comorbidities in various epidemiological studies. ${ }^{29}$ Previous studies showed that CDS can be reliably generated from electronic medical record data. ${ }^{27,28} \mathrm{CDS} \geq 7$ was associated with 5-fold increase in 1-year hospitalization risk. ${ }^{27}$ It was used in this study for matching cases with controls along with age, gender, race and ethnicity.

Symptom severity and overall illness trajectory were estimated on the basis of patients' self-reports. The behavioral profile included factors that may potentially affect hospitalization risk in older adults. Health literacy was assessed by self-report based on patient response to a Likert-type question about the level of personal illness comprehension. Medication adherence was assessed using the Medication Adherence scale which was shown to have excellent psychometric properties. ${ }^{30}$ Medication adherence self-efficacy was assessed using a validated questionnaire with known psychometric properties. ${ }^{31}$ Health literacy, medication adherence, and medication adherence self-efficacy were shown to be the major factors affecting efficacy of drug therapy in older adults with multiple chronic conditions $^{32}$ and were associated with urgent care utilization. ${ }^{33}$

\section{Pharmacogenetic profile}

Pharmacogenetic testing was performed by a New York State-licensed Personalized Medicine laboratory (GENETWORx, LLC) accredited by the College of American
Pathologists Laboratory Accreditation Program and with Clinical Laboratory Improvement Act certification. Among other tests, the laboratory carries out pharmacogenetic testing to detect common variants in genes which may affect individual response to medications. The GENETWORx Comprehensive PGRx Panel detects all common and many rare genetic variants with known clinical significance. Laboratory specimens are analyzed for the target genes using the GENETWORx Personalized Medicine Panel. The assay is based on the bead-based multiplex xTAG chemistry from Luminex Corporation. The CYP2D6 assay is an in vitro diagnostic assay from Luminex Corporation that also uses their xTag Technology. Genomic DNA is extracted from the submitted specimen and amplified by the polymerase chain reaction (PCR) using consensus oligonucleotide primers specific for the variants listed above. Genomic DNA is amplified in a multiplex fashion. The PCR is then subjected to a primer extension step that is specific for the allele that is being analyzed: Allele-Specific Primer Extension (ASPE). The 5' end of the ASPE primers is attached to an XTAG universal tag sequence. The 5' universal tag sequence is hybridized to the complementary anti-tag sequence coupled to a particular xMAP bead set that is then detected by the xMAP Luminex 200 analyzer.

The laboratory provides testing kits including instructional materials, consent forms, and buccal swab tools. After a buccal swab is collected from inside each cheek, it is placed in the provided envelopes and returned to the laboratory through overnight express mail. The testing results are provided by the testing facility through a password-protected secure online portal (www.GENETWORx.com) a week after the submission of the buccal swabs. The portal contains a detailed report with results of the genetic testing as well as interpretation of the findings. The portal also provided comprehensive education materials explaining the content of pharmacogenetic testing reports.

Pharmacogenetic profile was assessed on the basis of the presence or absence of mutant alleles responsible for the expression of proteins or enzymes affecting pharmacokinetics and pharmacodynamics of the prescribed medications. The gene panel tested in this study was chosen on the basis of the high prevalence of particular medication classes in older adults and evidence that sensitivity to these medications may be affected by known genetic polymorphisms. ${ }^{34,35}$ Variants in the following genes were tested in this study: CYP2C19, CYP2C9, VCORC1, CYP2D6, and CYP $3 A 4 / 3 A 5$. These variants were shown to play a major role in patient susceptibility to drugs frequently associated with ADEs in older adults with polypharmacy. ${ }^{5,36,37}$ 


\section{Statistical analysis}

Statistical analysis was carried out using the standard computer package IBM SPSS Statistics 22.0 (IBM Corporation, Somers, NY, USA, 2015). The results were presented as proportions for categorical variables and as means and standard deviations for continuous variables. Nonparametric tests were employed to compare the cases and controls since the normal distribution in the study population could not be assumed. ${ }^{38}$ The Wilcoxon signed-rank test was used while comparing the means of continuous variables from matched samples. The exact McNemar's test was used on paired nominal data. Statistical significance was confirmed at $p<0.05$. For analytical purposes, several variables were constructed on the basis of pharmacogenetic testing reports. Major pharmacogenetic polymorphism was defined as allele combination resulting in poor or rapid metabolizer status. A binary variable equal to 1 was introduced if at least one major genetic polymorphism was identified from the list of all tested genes, otherwise it was equal to zero. For gene-drug interaction analysis, a variable representing the number of major drug-gene interactions of moderate to serious severity was constructed for each study subject. The severity of drug-gene interactions indicated the degree of risk associated with the drug based on the patient's genetic status and was confirmed by an independent pharmacist review.

The study protocol was approved by Institutional Review Board at Columbia University Medical Center.

\section{Results \\ Sociodemographic profile}

Sociodemographic characteristics of the cases and controls are presented in Table 1. As cases and controls were matched by age group, gender, race, and ethnicity, there was no difference between these variables in the study groups. The average age of cases was $77 \pm 5$ years ranging from 69 to 83 years, and the average age of controls was $78 \pm 5$ years ranging from 70 to 84 years. The majority of the patients in both groups were single. All subjects indicated the presence of at least 1 caregiver, and only 1 subject in the control group lived alone. Most subjects were born in the US and reported good to excellent command of the English language. The annual income of the majority of the subjects was $<\$ 40,000$, and all the subjects were covered by Medicare insurance. Overall, no statistically significant difference in sociodemographic characteristics was found between the cases and controls.

\section{Clinical and behavioral profile}

The clinical and behavioral characteristics of the cases and controls are presented in Table 2. The CDS was used to match
Table I Sociodemographic characteristics of cases and controls

\begin{tabular}{lll}
\hline Parameters & Cases & Controls \\
\hline Age (mean $\pm \mathrm{SD}$ ), years & $77.2 \pm 5.0$ & $78.3 \pm 5 . \mathrm{I}$ \\
Gender (\%) & $\mathrm{M}=67 ; \mathrm{F}=33$ & $\mathrm{M}=67 ; \mathrm{F}=33$ \\
Race (\%) & $\mathrm{W}=83 ; \mathrm{B}=17$ & $\mathrm{~W}=83 ; \mathrm{B}=17$ \\
Ethnicity (\%) & $\mathrm{H}=67 ; \mathrm{NH}=33$ & $\mathrm{H}=67 ; \mathrm{NH}=33$ \\
Household size (mean $\pm \mathrm{SD})$ & $2.2 \pm 0.4$ & $2.0 \pm 0.6$ \\
Years in school (mean $\pm \mathrm{SD})$ & $10.3 \pm 2.7$ & $\mathrm{I} .2 \pm 5.5$ \\
Marital status (\%) & Married $=33 ;$ & Married $=33 ;$ \\
& single $=67$ & single $=67$ \\
Country of birth (\%) & $\mathrm{USA}=67 ;$ & $\mathrm{USA}=67 ;$ \\
& $\mathrm{non}-\mathrm{USA}=33$ & non-USA $=33$ \\
Full-time employment $(\%)$ & 0 & 0 \\
English proficiency $(\%)$ & $\mathrm{NP}=33 ; \mathrm{GE}=67$ & $\mathrm{NP}=33 ; \mathrm{GE}=67$ \\
Income (\%) & $<\$ 40 \mathrm{~K}=83 ;$ & $<\$ 40 \mathrm{~K}=83 ;$ \\
& $>\$ 40 \mathrm{~K}=17$ & $>\$ 40 \mathrm{~K}=17$ \\
Insurance $(\%)$ & $\mathrm{MM}=100 ;$ & $\mathrm{MM}=100 ;$ \\
& $\mathrm{PHP}=17$ & $\mathrm{PHP}=33$ \\
\hline
\end{tabular}

Abbreviations: $B$, black; $F$, female; GE, good to excellent; $H$, Hispanic; $M$, male; MM, Medicare/Medicaid; NH, non-Hispanic; NP, none to poor; PHP, private health plan; SD, standard deviation; $\mathrm{W}$, white.

Table 2 Clinical and behavioral characteristics of cases and controls

\begin{tabular}{|c|c|c|}
\hline Parameters & Cases & Controls \\
\hline $\begin{array}{l}\text { Chronic disease score } \\
(\text { mean } \pm \text { SD) }\end{array}$ & $7.8 \pm 1.2$ & $7.8 \pm 1.2$ \\
\hline $\begin{array}{l}\text { Medication adherence } \\
\text { score (mean } \pm S D)\end{array}$ & $6.9 \pm 1.1$ & $7.0 \pm 1.0$ \\
\hline $\begin{array}{l}\text { Medication adherence } \\
\text { self-efficacy (mean } \pm \text { SD) }\end{array}$ & $2.5 \pm 0.5$ & $2.7 \pm 0.4$ \\
\hline $\mathrm{BMI}($ mean $\pm \mathrm{SD})$ & $29.2 \pm 4.1$ & $29.3 \pm 3.1$ \\
\hline $\begin{array}{l}\text { Current alcohol } \\
\text { consumption (\%) }\end{array}$ & 0 & 0 \\
\hline Current smoking (\%) & 0 & 0 \\
\hline Former smoker (\%) & 33 & 67 \\
\hline $\begin{array}{l}\text { Number of comorbid } \\
\text { conditions (mean } \pm S D \text { ) }\end{array}$ & $8.2 \pm 1.2$ & $8.2 \pm 2.0$ \\
\hline $\begin{array}{l}\text { Number of geriatric } \\
\text { syndromes (mean } \pm \mathrm{SD} \text { ) }\end{array}$ & $4.0 \pm 1.7$ & $3.7 \pm 0.5$ \\
\hline Symptom severity (\%) & $\mathrm{NM}=17 ; \mathrm{MS}=83$ & $N M=33 ; M S=67$ \\
\hline Overall illness trajectory (\%) & $P=50 ; S=50 ; I=0$ & $P=50 ; S=33 ; I=17$ \\
\hline Health literacy (\%) & Insuf $=50 ;$ Suf $=50$ & Insuf $=50 ;$ Suf $=50$ \\
\hline $\begin{array}{l}\text { Number of medications } \\
\text { (mean } \pm S D \text { ) }\end{array}$ & $14.3 \pm 5.3$ & $14.0 \pm 2.9$ \\
\hline $\begin{array}{l}\text { Number of gene-drug } \\
\text { interactions (mean } \pm S D \text { ) }\end{array}$ & $2.8 \pm 2.2$ & $0 \pm 0^{\S}$ \\
\hline $\begin{array}{l}\text { Number of hospitalizations } \\
\text { in } 2 \text { years (mean } \pm S D \text { ) }\end{array}$ & $4.8 \pm 1.7$ & $0.2 \pm 0.4 \S$ \\
\hline $\begin{array}{l}\text { Number of hospitalizations } \\
\text { in } 5 \text { years (mean } \pm S D \text { ) }\end{array}$ & $11.2 \pm 9.3$ & $0.8 \pm 0.8 \S$ \\
\hline
\end{tabular}

Note: $\S^{S}$ tatistically significant difference between cases and controls $(P<0.05)$.

Abbreviations: BMI, body mass index; I, improving; Insuf, insufficient; MS, moderate to severe; NM, none to mild; P, progressing; S, stable; Suf, sufficient; SD, standard deviation

the cases and controls by patients' disease severity; the mean value was the same in both the groups: $7.8 \pm 1.2$. CDS $\geq 7$ indicated significant multi-morbidity ${ }^{26}$ in both the cases and controls. The mean number of chronic comorbid conditions 
was 8 and the mean number of geriatric syndromes was 4 in both the groups. Medication adherence in both the groups was similar: $6.9 \pm 1.1$ in cases and $7.0 \pm 1.0$ in controls. Medication adherence score $>6$ indicated appropriate adherence to medication regimen. ${ }^{30}$ Medication adherence self-efficacy score did not differ in both the groups and was $>2$ indicating sufficient patient confidence in their ability to take medications as prescribed. The mean number of prescribed medications in cases was $14.3 \pm 5.3$ ranging from 7 to 22 and that in the controls was $14.0 \pm 2.9$ ranging from 10 to 18 , indicating high level of polypharmacy in both the groups. Average BMI in both the groups was 29 with all the study subjects being overweight and 33\% cases and 50\% controls being obese. All the patients reported current abstinence from smoking and alcohol consumption. A higher proportion of cases $(83 \%)$ reported moderate to severe symptom severity as compared to controls $(67 \%)$. Overall illness trajectory was assessed as stable by $50 \%$ cases and as progressively worsening by another $50 \%$ of cases, whereas $17 \%$ of the controls reported improvement in their illness trajectory. There was a statistically significant difference in the number of hospitalizations between cases and controls $(p<0.05)$. The average number of hospitalizations in the last 2 years in cases was $4.8 \pm 1.7$ ranging from 3 to 7 and that in the controls was $0.2 \pm 0.4$ ranging from 0 to 1 . The average number of hospitalizations in the last 5 years in cases was $11.2 \pm 9.3$ ranging from 3 to 23 , whereas in controls it was $0.8 \pm 0.8$ ranging from 0 to 2 .

\section{Pharmacogenetic profile}

The results of pharmacogenetics testing are listed in Table 3. Each case was found to have at least one major pharmacogenetic polymorphism defined as mutant allele combination resulting in poor or rapid metabolizer status. No major pharmacogenetic polymorphisms were found in the controls. Based on the exact McNemar's test, the difference in major pharmacogenetic polymorphism frequency between the cases and controls was statistically significant $(p<0.05)$. In $50 \%$ of the cases, $>1$ major pharmacogenetic polymorphism was found. The frequency of $C Y P 2 C 19$ rapid metabolizer, CYP3A4/5 poor metabolizer, VKORC1 low sensitivity, and $C Y P 2 D 6$ rapid metabolizer status in the cases was $67 \%$, $33 \%, 33 \%$, and $17 \%$, respectively, whereas in the general US population, it was reported not to exceed $4.5 \%, 10 \%$, $12 \%$, and $4.3 \%$, respectively. ${ }^{14}$ The mean number of major gene-drug interactions in the cases was $2.8 \pm 2.2$, whereas no major drug-gene interactions were identified in the controls. The difference in the number of major drug-gene interactions between the cases and controls was statistically significant $(p<0.05)$ based on the Wilcoxon signed-rank test.
Table 3 Results of pharmacogenetic testing

\begin{tabular}{|c|c|c|c|c|c|}
\hline \multirow{2}{*}{$\begin{array}{l}\text { Hospital } \\
\text { admissions }\end{array}$} & \multicolumn{5}{|c|}{ Pharmacogenetic profile } \\
\hline & CYP2CI9 & CYP2C9 & VKORCI & CYP2D6 & CYP3A4/5 \\
\hline & \multicolumn{5}{|l|}{ Cases } \\
\hline High & ENM & ENM & HWS & ENM & PM \\
\hline High & ENM & ENM & IWS & RM & ENM \\
\hline High & RM & ENM & IWS & ENM & PM \\
\hline High & RM & ENM & NWS & ENM & ENM \\
\hline High & RM & ENM & HWS & ENM & ENM \\
\hline \multirow[t]{2}{*}{ High } & RM & ENM & IWS & ENM & IM \\
\hline & \multicolumn{5}{|l|}{ Controls } \\
\hline Low & ENM & ENM & NWS & ENM & ENM \\
\hline Low & IM & ENM & NWS & IM & ENM \\
\hline Low & IM & ENM & NWS & ENM & ENM \\
\hline Low & IM & ENM & NWS & ENM & ENM \\
\hline Low & IM & ENM & NWS & IM & ENM \\
\hline Low & IM & $\mathbb{I M}$ & IWS & ENM & ENM \\
\hline
\end{tabular}

Abbreviations: ENM, extensive/normal metabolizer; HWS, high warfarin sensitivity; IM, intermediate metabolizer; IWS, increased warfarin sensitivity; NWS, normal warfarin sensitivity; PM, poor metabolizer; RM, rapid metabolizer.

\section{Discussion}

The results of this study demonstrated that there is an association between high hospitalization rates and pharmacogenetic polymorphism in older adults with polypharmacy. This is the first study that systematically explored whether pharmacogenetic polymorphism is an independent risk factor for $\mathrm{FH}$ in older adults with polypharmacy. In this study, a nested case-control design was used to account for potential covariates. The nested case-control design is widely used in studies of biologic precursors, such as somatic mutations, especially when assessment of exposure requires costly and/ or labor-intensive testing. ${ }^{39}$ By matching on factors such as age, race, gender, and comorbidity score, and selecting controls from a relevant cohort, the nested case-control design is generally considered to be efficient in addressing potential study biases. ${ }^{40}$

The results of this study support broader introduction of pharmacogenetic testing in the care of older adults. Based on a 2005-2006 survey study, $>36 \%$ of older adults were taking at least 5 prescription medications. ${ }^{41}$ In older adults, polypharmacy has been associated with increased health care costs, adverse drug reactions, drug interactions, medication nonadherence, impaired functional and cognitive status, falls, urinary incontinence, and malnutrition. ${ }^{5}$ Not surprisingly, polypharmacy and potentially inappropriate medication use were shown to be a significant precipitating factor in frequent hospital admissions. ${ }^{42}$ The pharmacogenetic information that may currently be made available to guide therapy selection and adjust dose requirements can significantly enhance the utility of prescription drugs and provides an opportunity for improving patient care. ${ }^{19}$ 
Additionally, this new technology can lead to a decrease in the number of ADEs and the enormous costs associated with these iatrogenic hospitalizations and emergency room visits. ${ }^{43}$ Recent reports demonstrated that there is a significant overlap between classes of medications most frequently implicated as cause of ADE in older adults ${ }^{36,44}$ and classes of medications, action of which is significantly affected by pharmacogenetic polymorphisms. ${ }^{10}$ These classes include anticoagulants, $\beta$-blockers, calcium-channel blockers, nonsteroidal anti-inflammatory drugs, antibiotics, antidepressants, benzodiazepines, proton pump inhibitors, and chemotherapeutic agents. ${ }^{10,36}$ Medications from the same classes are listed among most frequently prescribed drugs for older adults. ${ }^{45}$ Thus, higher level of polypharmacy makes it more likely for a patient to be affected by potential drug-gene interactions.

Several recent reports supported the potential value of pharmacogenetic testing in older adults with polypharmacy. ${ }^{46}$ In a recent observational study, a prospective cohort of older adults whose medication regimen was optimized according to pharmacogenetic testing was compared to a propensity score-matched historical cohort. ${ }^{23}$ Comparison of urgent care utilization between these two cohorts showed that patients treated according to pharmacogenetics-guided treatment plan had a significant decrease in hospitalizations and emergency department visits with average cost savings of \$218. A recent article reviewed a case of a 74-year-old woman with polypharmacy hospitalized due to severe ADE. ${ }^{43}$ Pharmacogenetic testing in this patient established major genetic CYP3A5 polymorphism. Ensuing pharmacogenetic-guided medication regimen readjustment led to remarkable patient recovery with complete remission of coetaneous symptoms and control of cardiac, hepatic, and renal symptomatology. In another study, application of pharmacogenetics-guided recommendations in older adults with polypharmacy in a long-term care facility ${ }^{9}$ resulted in medication optimization for $50 \%$ of the polypharmacy population tested with an estimated annual savings of $\$ 621$ per patient.

As the cost-effectiveness of pharmacogenetic testing is considered one of the major barriers toward its wide introduction to clinical practice, ${ }^{47}$ these recent results uncover potential approaches for overcoming cost barriers for pharmacogenetics by targeting subgroups of patients for whom pharmacogenetic testing may result in substantial cost savings such as older adults with polypharmacy. Further studies with appropriate design and sample size are necessary to identify the prevalence of pharmacogenetic polymorphisms in frequently hospitalized older adults and systematically test the hypothesis that pharmacogenetic polymorphism is an independent risk factor of FH in older adults with polypharmacy. Effective optimization of medication regimen in older adults with polypharmacy requires a fundamental shift in clinical decision making from the "one gene - one drug - one disease" paradigm to a "pharmacogenetic profile polypharmacy - multimorbidity" paradigm.

The ultimate goal of this study is to develop comprehensive evidence-based clinical decision support technologies facilitating effective introduction of pharmacogenetics into routine clinical care of older adults. Identifying priority populations of older adults who are most likely to benefit from pharmacogenetic testing is a crucial step toward accomplishing this goal. This study provided initial evidence that pharmacogenetic polymorphism is potentially an independent risk factor for high hospitalization rates in older adults. Definitive confirmation of this hypothesis will require that a definitive randomized clinical trial (RCT) be performed to demonstrate that pharmacogenetic testing in frequently hospitalized older adults with polypharmacy results in reduction of urgent care utilization, decreased health care costs, and improved quality of care. As lack of convincing evidence demonstrating that genetic testing improves clinical outcomes precludes Medicare from covering this procedure in older adults, RCT-level evidence may greatly contribute to supporting insurance coverage of pharmacogenetic testing in frequently hospitalized older adults with polypharmacy.

Pharmacogenetic testing has an enormous potential in optimizing drug therapy and improving quality of care. A plethora of pharmacogenetic studies over recent years have resulted in a significant body of knowledge on genetic-based variations affecting individual drug susceptibility. However, the application of pharmacogenetics in routine patient care is hampered by conflicting information concerning its clinical utility in specific patient subgroups and lack of consensus on the extent to which physicians are expected to act on pharmacogenetic test results. The majority of previous pharmacogenetic studies of clinical utility have focused on the associations between specific genes and a single drug or disease. Our study introduced an innovative approach in assessing the potential cumulative health care utilization impact of pharmacogenetic profile in older adults with multiple comorbidities who have been prescribed a large number of medications. Thus, this study is characterized by several important strengths. First, this study introduces an important shift in the application of pharmacogenetic testing from a "one gene - one drug - one disease" approach to a "pharmacogenetic profile - polypharmacy - multimorbidity" approach. This approach is particularly important in the context of older adults because it provides systematic 
evidence on the potential utility of pharmacogenetic testing in older adults routinely seen in inpatient and outpatient care. There is a high probability that at least one medication may be affected by genetic polymorphisms in these patients because the metabolism, transport, or action of the drug is affected by different enzymes. Drug efficacy and ADEs can also be affected by the natural aging process and the presence of geriatric syndromes. Second, the study's specific focus on older adults with polypharmacy who are frequently admitted to hospital is innovative. Limited systematic evidence exists on how important it is to perform pharmacogenetic testing in such a vulnerable subgroup as frequently hospitalized older adults with complex medication regimens and multiple comorbidities. Evidence of higher prevalence of pharmacogenetic polymorphism in this subgroup may be of major importance because it may lead to recommendations to prioritize genetic testing in these patients to optimize drug therapy and prevent frequent readmissions. Third, this study utilized a nested case-control design to systematically compare the frequency of pharmacogenetic polymorphisms in frequently hospitalized older adults with polypharmacy with matched individuals who did not have a history of frequent hospital admissions. This study was designed to identify whether pharmacogenetic polymorphisms in older adults with polypharmacy is an independent risk factor for FH. No systematic study has been conducted that was aimed at addressing this question using a nested case-control design that accounts for multiple hospitalization risk factors. Fourth, the proposed study establishes an evidence-based approach for targeted application of pharmacogenetic testing in older adults with polypharmacy. Systematic evidence supporting pharmacogenetic testing in frequently hospitalized older adults with polypharmacy may lead to targeted testing in a group of patients who are most likely to benefit from the testing results. With sufficient evidence, the hospital staff may be advised to incorporate pharmacogenetic testing of this specific cohort into the routine hospital discharge workflow and utilize the pharmacogenetic testing results to optimize the medication regimen on discharge as a part of a predischarge medication reconciliation program. This approach may potentially reduce readmissions in these patients, improve their quality of care, and reduce their health care expenses. Targeting particular patient subgroups may maximize the potential benefits from pharmacogenetic testing and may promote the use of this promising technique in a cost-effective way. Future intervention trials built on the evidence provided in this study will be able to definitively test the clinical impact and cost-effectiveness of pharmacogenetics-guided care in older adults.
The study has several limitations including small sample size and lack of sufficient racial heterogeneity. Small sample size precluded us from estimating relative risks and contributions of different factors to hospitalization risk using conditional logistic regression. The Wilcoxon signed-rank test was used in this study to compare the means of continuous variables from matched samples because it does not assume normal distribution for dependent samples. ${ }^{48}$ The exact McNemar's test was used on paired nominal data in this study because it utilizes conservative estimates applicable for small samples. ${ }^{49}$ These two tests were used for small sample comparisons in previous studies; however, the difference between samples should be very substantial to achieve an acceptable level of statistical significance. ${ }^{50,51}$ Although recent studies concluded that there are no principal objections to using appropriate statistical tests for groups with small sample sizes, ${ }^{50-52}$ no definitive conclusions can be adequately drawn from our proof-of-concept study due to its limited sample size. However, even with small sample size, this study was able to demonstrate potential difference in the frequency of pharmacogenetic polymorphisms in older adults with polypharmacy who are frequently hospitalized versus those who are rarely admitted to a hospital. Our results support the implementation of more comprehensive study with sufficient power to account for multiple hospitalization risk factors and racial/ethnic heterogeneity. Future study should employ larger sample size that allows for population-based genetic analysis including analysis of Hardy-Weinberg equilibrium. ${ }^{53}$ As polypharmacy affects not only older adults but also people with human immunodeficiency, ${ }^{54,55}$ cancer, ${ }^{56,57}$ and other complex conditions, ${ }^{58}$ identification of utility of pharmacogenetic testing ${ }^{59}$ in other patient subgroups with polypharmacy may help identify priority populations for medication optimization using pharmacogenetic testing.

\section{Conclusion}

In a pilot nested case-control study, frequency of pharmacogenetic polymorphisms was shown to be significantly higher in frequently hospitalized older adults with polypharmacy as compared to older adults with polypharmacy rarely admitted to a hospital. Due to a small sample size, definitive conclusions could not be drawn; however, the results of this study justify conducting a large-scale population-based study. Future research should provide definitive evidence to support a recommendation for providing pharmacogenetic testing to all older adults with polypharmacy who are frequently admitted to a hospital. This recommendation would facilitate broad introduction of a personalized medication regimen, potentially reducing hospitalization rates, decreasing health 
care expenditures, and improving quality of care and patient quality of life in such a vulnerable population as older adults with polypharmacy.

\section{Acknowledgment}

This work was partially supported by funding from the National Institutes of Health (R01LM010016 and U01HG008680).

\section{Disclosure}

The authors report no conflicts of interest in this work.

\section{References}

1. Murad K, Kitzman DW. Frailty and multiple comorbidities in the elderly patient with heart failure: implications for management. Heart Fail Rev. 2012;17(4-5):581-588.

2. Russo CA, Elixhauser A. Hospitalizations in the Elderly Population, 2003. Statistical Brief\#6. May 2006. Agency for Healthcare Research and Quality, Rockville, MD. Available from: https://www.hcup-us.ahrq. gov/reports/statbriefs/sb6.jsp. Accessed October 4, 2016.

3. Fillenbaum GG, Pieper CF, Cohen HJ, Cornoni-Huntley JC, Guralnik JM. Comorbidity of five chronic health conditions in elderly community residents: determinants and impact on mortality. J Gerontol A Biol Sci Med Sci. 2000;55(2):M84-M89.

4. Franchi C, Nobili A, Mari D, et al; REPOSI Investigators. Risk factors for hospital readmission of elderly patients. Eur J Intern Med. 2013;24(1):45-51.

5. Maher RL, Hanlon J, Hajjar ER. Clinical consequences of polypharmacy in elderly. Expert Opin Drug Saf. 2014;13(1):57-65.

6. Slabaugh SL, Maio V, Templin M, Abouzaid S. Prevalence and risk of polypharmacy among the elderly in an outpatient setting: a retrospective cohort study in the Emilia-Romagna region, Italy. Drugs Aging. 2010;27(12):1019-1028.

7. Sganga F, Landi F, Ruggiero C, et al. Polypharmacy and health outcomes among older adults discharged from hospital: results from the CRIME study. Geriatr Gerontol Int. 2015;15(2):141-146.

8. Marcum ZA, Amuan ME, Hanlon JT, Aspinall SL, Handler SM, Ruby CM, Pugh MJ. Prevalence of unplanned hospitalizations caused by adverse drug reactions in older veterans. J Am Geriatr Soc. 2012;60(1):34-41.

9. Saldivar JS, Taylor D, Sugarman EA, Cullors A, Garces JA, Oades K, Centeno J. Initial assessment of the benefits of implementing pharmacogenetics into the medical management of patients in a long-term care facility. Pharmgenomics Pers Med. 2016;9:1-6.

10. Hiratsuka M, Sasaki T, Mizugaki M. Genetic testing for pharmacogenetics and its clinical application in drug therapy. Clin Chim Acta. 2006;363(1-2):177-186.

11. Oetjens MT, Denny JC, Ritchie MD, et al. Assessment of a pharmacogenomic marker panel in a polypharmacy population identified from electronic medical records. Pharmacogenomics. 2013;14(7):735-744.

12. Leeder JS. Pharmacogenetics and pharmacogenomics. Pediatr Clin North Am. 2001;48(3):765-781.

13. Li J, Bluth MH. Pharmacogenomics of drug metabolizing enzymes and transporters: implications for cancer therapy. Pharmgenomics Pers Med. 2011;4:11-33.

14. Wijnen PA, Op den Buijsch RA, Drent M, et al. Review article: the prevalence and clinical relevance of cytochrome $\mathrm{P} 450$ polymorphisms. Aliment Pharmacol Ther. 2007;26(Suppl 2):211-219.

15. Cavallari LH, Jeong H, Bress A. Role of cytochrome P450 genotype in the steps toward personalized drug therapy. Pharmgenomics Pers Med. 2011;4:123-136.

16. García-González X, Cabaleiro T, Herrero MJ, McLeod H, LópezFernández LA. Clinical implementation of pharmacogenetics. Drug Metab Pers Ther. 2016;31(1):9-16.
17. Sehrt D, Meineke I, Tzvetkov M, Gültepe G, Brockmöller J. Carvedilol pharmacokinetics and pharmacodynamics in relation to CYP2D6 and ADRB pharmacogenetics. Pharmacogenomics. 2011;12(6):783-795.

18. Brown JT, Abdel-Rahman SM, van Haandel L, Gaedigk A, Lin YS, Leeder JS. Single dose, CYP2D6 genotype-stratified pharmacokinetic study of atomoxetine in children with ADHD. Clin Pharmacol Ther. 2016;99(6):642-650.

19. Sim SC, Ingelman-Sundberg M. Pharmacogenomic biomarkers: new tools in current and future drug therapy. Trends Pharmacol Sci. 2011;32(2):72-81.

20. Stergiopoulos K, Brown DL. Genotype-guided vs clinical dosing of warfarin and its analogues: meta-analysis of randomized clinical trials. JAMA Intern Med. 2014;174(8):1330-1338.

21. Finkelstein J, Friedman C, Hripcsak G, Cabrera M. Potential utility of precision medicine for older adults with polypharmacy: a case series study. Pharmgenomics Pers Med. 2016;9:31-45.

22. Chaudhry SI, McAvay G, Chen S, Whitson H, Newman AB, Krumholz HM, Gill TM. Risk factors for hospital admission among older persons with newly diagnosed heart failure: findings from the Cardiovascular Health Study. J Am Coll Cardiol. 2013;61(6):635-642.

23. Brixner D, Biltaji E, Bress A, et al. The effect of pharmacogenetic profiling with a clinical decision support tool on healthcare resource utilization and estimated costs in the elderly exposed to polypharmacy. J Med Econ. 2016;19(3):213-228.

24. Fortinsky RH, Madigan EA, Sheehan TJ, Tullai-McGuinness S, Kleppinger A. Risk factors for hospitalization in a national sample of medicare home health care patients. J Appl Gerontol. 2014;33(4):474-493.

25. Von Korff M, Wagner EH, Saunders K. A chronic disease score from automated pharmacy data. J Clin Epidemiol. 1992;45(2):197-203.

26. Clark DO, Von Korff M, Saunders K, Baluch WM, Simon GE. A chronic disease score with empirically derived weights. Med Care. 1995;33(8): 783-795.

27. Johnson RE, Hornbrook MC, Nichols GA. Replicating the chronic disease score (CDS) from automated pharmacy data. J Clin Epidemiol. 1994;47(10):1191-1199.

28. Schneeweiss S, Seeger JD, Maclure M, Wang PS, Avorn J, Glynn RJ. Performance of comorbidity scores to control for confounding in epidemiologic studies using claims data. Am J Epidemiol. 2001;154(9):854-864.

29. Schneeweiss S, Maclure M. Use of comorbidity scores for control of confounding in studies using administrative databases. Int J Epidemiol. 2000;29(5):891-898.

30. Morisky DE, Green LW, Levine DM. Concurrent and predictive validity of a self-reported measure of medication adherence. Med Care. 1986;24(1):67-74.

31. Fernandez S, Chaplin W, Schoenthaler AM, Ogedegbe G. Revision and validation of the medication adherence self-efficacy scale (MASES) in hypertensive African Americans. J Behav Med. 2008;31(6):453-462.

32. Nair NP, Chalmers L, Peterson GM, Bereznicki BJ, Castelino RL, Bereznicki LR. Hospitalization in older patients due to adverse drug reactions - the need for a prediction tool. Clin Interv Aging. 2016;11:497-505.

33. García-Pérez L, Linertová R, Lorenzo-Riera A, Vázquez-Díaz JR, DuqueGonzález B, Sarría-Santamera A. Risk factors for hospital readmissions in elderly patients: a systematic review. QJM. 2011;104(8):639-651.

34. Seripa D, Paroni G, Urbano M, Santini SA, D’Onofrio G, Pilotto A. Pharmacogenetics in older people: what we know and what we need to know. J Nephrol. 2012;25(Suppl 19):S38-S47.

35. Seripa D, Panza F, Daragjati J, Paroni G, Pilotto A. Measuring pharmacogenetics in special groups: geriatrics. Expert Opin Drug Metab Toxicol. 2015;11(7):1073-1088.

36. Hohl CM, Dankoff J, Colacone A, Afilalo M. Polypharmacy, adverse drug-related events, and potential adverse drug interactions in elderly patients presenting to an emergency department. Ann Emerg Med. 2001;38(6):666-671.

37. Campbell NL, Skaar TC, Perkins AJ, Gao S, Li L, Khan BA, Boustani MA. Characterization of hepatic enzyme activity in older adults with dementia: potential impact on personalizing pharmacotherapy. Clin Interv Aging. 2015;10:269-275. 
38. Breslow NE, Day N. Statistical Methods in Cancer Epidemiology: Analysis of Case-Control Studies, Volume I. Lyon, France: IARC Scientific Publications.

39. Wang Y, McCullough ML, Stevens VL, et al. Nested case-control study of energy regulation candidate gene single nucleotide polymorphisms and breast cancer. Anticancer Res. 2007;27(1B):589-593.

40. Ernster VL. Nested case-control studies. Prev Med. 1994;23(5):587-590.

41. Qato DM, Alexander GC, Conti R, et al. Use of prescription and overthe-counter medications and dietary supplements among older adults in the United States. JAMA. 2008;300:2867-2878.

42. Sehgal V, Bajwa SJ, Sehgal R, Bajaj A, Khaira U, Kresse V. Polypharmacy and potentially inappropriate medication use as the precipitating factor in readmissions to the hospital. J Fam Med Prim Care. 2013;2(2): 194-199.

43. Isidoro-García M, Sánchez-Martín A, García-Berrocal B, Román-Curto C. Primun non nocere, polypharmacy and pharmacogenetics. Pharmacogenomics. 2015;16(17):1903-1905.

44. Salvi F, Marchetti A, D’Angelo F, Boemi M, Lattanzio F, Cherubini A. Adverse drug events as a cause of hospitalization in older adults. Drug Saf. 2012;35(Suppl 1):29-45.

45. Moxey ED, O’Connor JP, Novielli KD, Teutsch S, Nash DB. Prescription drug use in the elderly: a descriptive analysis. Health Care Financ Rev. 2003;24(4):127-141.

46. Nightingale G, Hajjar E, Swartz K, Andrel-Sendecki J, Chapman A. Evaluation of a pharmacist-led medication assessment used to identify prevalence of and associations with polypharmacy and potentially inappropriate medication use among ambulatory senior adults with cancer. J Clin Oncol. 2015;33(13):1453-1459.

47. Shah RR, Gaedigk A, LLerena A, Eichelbaum M, Stingl J, Smith RL. CYP450 genotype and pharmacogenetic association studies: a critical appraisal. Pharmacogenomics. 2016;17(3):259-275.

48. Klotz J. Small sample power and efficiency for the one sample Wilcoxon and normal scores tests. Ann Math Statist. 1963;34(2):624-632.
49. Fagerland MW, Lydersen S, Laake P. The McNemar test for binary matched-pairs data: mid-p and asymptotic are better than exact conditional. BMC Med Res Methodol. 2013;13:91.

50. Hoyle R, Editor. Statistical Strategies for Small Sample Research. New York: SAGE Publications; 1999.

51. Pett MA. Nonparametric Statistics for Health Care Research: Statistics for Small Samples and Unusual Distributions. New York: SAGE Publications; 1997.

52. De Winter JCF. Using the Student's t-test with extremely small sample sizes. Prac Assess Res Eval. 2013;18(10):1-12.

53. Wigginton J, Cutler D, Abecasis G. A note on exact tests of HardyWeinberg equilibrium. Am J Hum Genet. 2005,76(5):887-893.

54. Gimeno-Gracia M, Crusells-Canales MJ, Armesto-Gómez FJ, Compaired-Turlán V, Rabanaque-Hernández MJ. Polypharmacy in older adults with human immunodeficiency virus infection compared with the general population. Clin Interv Aging. 2016;11:1149-1157.

55. Holtzman C, Armon C, Tedaldi E, Chmiel JS, Buchacz K, Wood K, Brooks JT; the HOPS Investigators. Polypharmacy and risk of antiretroviral drug interactions among the aging HIV-infected population. J Gen Intern Med. 2013;28(10):1302-1310.

56. Sirachainan E, Jaruhathai S, Trachu N, et al. CYP2D6 polymorphisms influence the efficacy of adjuvant tamoxifen in Thai breast cancer patients. Pharmgenomics Pers Med. 2012;5:149-153.

57. Woopen H, Richter R, Ismaeel F, Chekerov R, Roots I, Siepmann T, Sehouli J. The influence of polypharmacy on grade III/IV toxicity, prior discontinuation of chemotherapy and overall survival in ovarian cancer. Gynecol Oncol. 2016;140(3):554-558.

58. Franssen FM, Spruit MA, Wouters EF. Determinants of polypharmacy and compliance with GOLD guidelines in patients with chronic obstructive pulmonary disease. Int J Chron Obstruct Pulmon Dis. 2011;6:493-501.

59. Carpenter JS, Rosenman MB, Knisely MR, Decker BS, Levy KD, Flockhart DA. Pharmacogenomically actionable medications in a safety net health care system. SAGE Open Med. 2016;4:2050312115624333.
Pharmacogenomics and Personalized Medicine

\section{Publish your work in this journal}

Pharmacogenomics and Personalized Medicine is an international, peerreviewed, open access journal characterizing the influence of genotype on pharmacology leading to the development of personalized treatment programs and individualized drug selection for improved safety, efficacy and sustainability. This journal is indexed on the American Chemical

\section{Dovepress}

Society's Chemical Abstracts Service (CAS). The manuscript management system is completely online and includes a very quick and fair peer-review system, which is all easy to use. Visit http://www.dovepress. $\mathrm{com} /$ testimonials.php to read real quotes from published authors. 